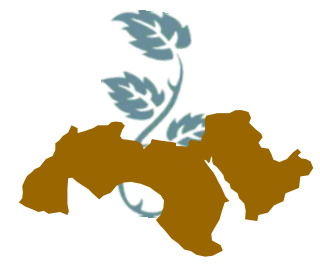

Arab Univ.

J. Agric. Sci., Ain Shams Univ., Cairo, 24(2), 623-632, 2016

\title{
EFFECT OF IRRIGATION SCHEDULING AND PLANT DENSITY ON PRODUCTIVITY AND QUALITY OF STRAWBERRY GROWN IN WATER CULTURE
}

\author{
Enass N. Eissa ${ }^{1}$, U.A. El-Behairy ${ }^{2}$, Zaki Lashine ${ }^{2}$ and S.H. Ahmed ${ }^{1}$ \\ 1. Agricultural Research Center (ARC), Giza, Egypt \\ 2. Horticulture Department, Fac. of Agric., Ain Shams University, Cairo, Egypt
}

Keywords: Soilless culture, NFT, Strawberry

\section{ABSTRACT}

The experiment was carried out at the experimental site of Arid Land Agricultural graduate studies and Research Institute (ALARI), Faculty of Agriculture, Ain Shams University, Qalyobia Governorate during the two successive seasons of 2013/2014 and 2014/2015. Fresh bare root strawberry (Fragaria $x$ ananassa) transplants cv. Festival were used in this experiment. The aim of the study was to determine the best irrigation scheduling and better strawberry density to get the best production and quality of strawberry fruits grown in A-shape NFT system. Five irrigation schedules (24 hours (control), 1/4 / 1/2 hour (Sched 1), $1 / 4 / 1$ hour (Sched 2), $1 / 4 / 2$ hours (Sched 3), $1 / 4$ / 3 hours (Sched 4) and two plant density per square meter (21 plants / $\mathrm{m}^{2}$ "PD-1",32 plants $/ \mathrm{m}^{2}$ "PD-2") and the combinations among them were tested in this experiment. The EC of the nutrient solution was adjusted at the range of $2.0-2.5$ m. hhos $^{-2}$ and $\mathrm{pH}$ was maintained at the range of 5.5-6.0 throughout the experimental time. The measurements recorded were as follows: number of leaves, \% of fruit set, early and total yield and mean fruit weight. Quality measurements included total soluble solids (TSS) (the percentage of TSS was determined by using hand refractometer), ascorbic acid (vitamin C), and fruit acidity. Results indicated that irrigating plants for 15 min every $1 / 2$ hour increased productivity (mean fruit weight, early yield and total yield) and quality (TSS) but reduced vitamin $\mathrm{C}$ and fruit acidity of strawberry.
While the irrigating plants every 1 hour reduced number of leaves, set \%, early \& total yield, mean fruit weight, TSS.

\section{INTRODUCTION}

Strawberry (Fragaria $\times$ ananassa) is a perennial, low-creeping, stoloniferous herb belonging to the family Rosaceae. It is basically a temperate fruit crop, widely distributed due to its genotypic diversity, high heterozygous nature and broad range of environmental adaptations (Sharma and Sharma, 2004). It is perishable crop which is exceedingly in demand for its taste, profitability, high yield and good quality. It has a unique, highly desirable taste and flavor and is one of the most popular fruits around the world (Sturnm et al 2003). It is a rich source of vitamins and minerals with delicate flavors (Sharma, 2002). It also contains a higher percentage of phenolic and flavonoids (Hakkinen and Torronen, 2000).

Strawberry is one of the most important crops for export in Egypt, and is produced in open fields, with heavy harvesting occurring mostly in the winter months of the year. This allows Egyptians strawberry growers to take advantage of the high fruit prices when other states do not have production due to cold weather. In spite of this competitive advantage, increasing competition from international markets has prompted growers to seek alternative production systems that could enhance crop yield and quality, as well as fruit earliness. Soilless culture may be an alternative to fulfill those goals. 
Soilless plant production has been practiced for several millennia and it permits crops to be grown where no suitable soil exists or where the soil is contaminated in some manner or other. Maximum yields are possible and this makes the system economically feasible in high-density and expensive land areas. According to Takeda (2000), future growth of soilless culture will depend on the development of production systems and substrates that are competitive in costs and returns with conventional agriculture.

Poor timing or insufficient irrigation could result in crop stress and reduced yields, whereas excessive watering may diminish yield and quality and increase the risk of nutrient leaching (Simonne and Dukes, 2009). Kruger et al (1999) and Kirnak et al (2003) showed a positive influence of proper irrigation on strawberry yield, fruit size and quality in comparison to non-irrigated plants. Another report found that there is a significant effect of irrigation on strawberry flavor (Hoberg et al 2002).

Planting density plays an important role in achieving high productivity per unit area. High planting density resulted in highest yield, largest berries, and the best quality fruit (Petersen, 1998). In strawberry, marketable yields were higher at narrower spacing than wider spacing (Legard et al 2000). Growth and yield per plant increased by increasing plant spacing from 20 to $30 \mathrm{~cm}$ and also resulted in a greater leaf area and leaf area index, but the highest harvest index and yield per square meter were obtained at the closest spacing (DeCamacaro et al 2004). Planting density also greatly influence production and fruit quality of strawberry plants that are grown from cuttings (Jansen, 1997). Improper selection of plant population density limits the productivity and acreage of strawberry.

Studies were conducted to see the impact of irrigation scheduling and plant densities on yield and quality of strawberry fruits in strawberry plants grown in A-shape NFT system.

\section{MATERIALS AND METHODS}

The experiment was carried out at experimental site of Arid Land Agricultural graduate studies and Research Institute (ALARI), Faculty of Agriculture, Ain Shams University, Qalyobia Governorate during the two successive seasons of $2013 / 2014$ and $2014 / 2015$. The study was conducted to determine the best irrigation scheduling and better strawberry density to get the best pro- duction and quality of strawberry fruits in strawberry plants grown in A-shape NFT system.

Fresh bare root strawberry (Fragaria $x$ ananassa) transplants Cv. Festival were used in this experiment. Transplants were transferred to their final place inside the holes of PVC pipes on 1/9/2013 and $3 / 9 / 2014$ in both seasons respectively.

The NFT system was used as a water culture system in this experiment.The NFT system "Ashape system" consists of triangle iron frame forming about 60 degrees triangle. PVC pipe (4 inch) worked as gullies fixed on this frames on the height of $40 \mathrm{~cm}$ from the soil surface to perform the bottom level, $25 \mathrm{~cm}$ from the bottom level to perform the middle level and $25 \mathrm{~cm}$ from the middle level to perform the top level. Plants were housed in holes preformed in the PVC pipe.

The nutrient solution described by El-Behairy (1994) Table (1) was used in this experiment. The Electrical Conductivity (EC) was adjusted at the range of 2.0-2.5 m.mhos ${ }^{-2}$ and $\mathrm{pH}$ was maintained at the range of 5.5-6.0 throughout the experimental time. System was irrigated from tank filled with diluted nutrient solution. Diluted nutrient solution was withdrew by 48 watt submersible pump via 18 $\mathrm{mm}$ irrigation pipe to lateral pipes $4 \mathrm{~mm}$, connected to the upper end of the NFT gutter. The nutrient solution was collected from each gutter by a plastic funnel fixed in the lower end of the gutter. The nutrient solution was switched on according each treatment but for 15 minutes in each time.

Table 1. Chemical composition of the used nutrient solution

\begin{tabular}{|c|c|}
\hline Elements & Concentrations (ppm) \\
\hline $\mathrm{N}$ & 250 \\
$\mathrm{P}$ & 35 \\
$\mathrm{~K}$ & 350 \\
$\mathrm{Ca}$ & 180 \\
$\mathrm{Mg}$ & 50 \\
$\mathrm{Fe}$ & 3 \\
$\mathrm{Mn}$ & 1 \\
$\mathrm{Cu}$ & 0.1 \\
$\mathrm{Zn}$ & 0.06 \\
$\mathrm{~B}$ & 0.1 \\
$\mathrm{Mo}$ & 0.01 \\
\hline
\end{tabular}



strawberry grown in water culture

The treatments included two factors; the main factor was the irrigation schedule, five irrigation schedule have been tested in relation to production and quality of strawberry as follow (Control (24 hours), Sched $1(1 / 4 / 1 / 2$ hour), Sched $2(1 / 4 / 1$ hour), Sched 3 ( $1 / 4 / 2$ hours), Sched 4 ( $1 / 4 / 3$ hours) and the sub main factor was the plant density, two plant densities per square meter have been tested in this experiment as follow (21 plants / $\mathrm{m}^{2}$ "PD-1", 32 plants $/ \mathrm{m}^{2}$ "PD-2").

Five irrigation schedules and two plant densities per square meter were tested to perform 10 interactions as follows:

1. 24 hours + PD-1 (control 1)

2. 24 hours + PD-2 (control 2)

3. Sched $1+\mathrm{PD}-1$

4. Sched 1+PD-2

5. Sched 2+ PD-1

6. Sched 2+ PD-2

7. Sched 3+PD-1

8. Sched 3+ PD-2

9. Sched 4+ PD-1

10. Sched 4+ PD-2

The Electrical Conductivity (EC) was adjusted at the range of 2.0-2.5 m.mhos ${ }^{-2}$ and $\mathrm{pH}$ was maintained at the range of 5.5-6.0 throughout the experimental time.

The measurements recorded were as follows: Vegetative growth measurements include number of leaves. Productivity measurements include set $\%$, early yield, total yield and mean fruit weight. Quality measurements include total soluble solids (TSS) were recorded as (\%) using hand refractometer (Atago No.1, 0-32 Brix, Japan), ascorbic acid (vitamin C) was determined in fruit juice as described in A.O.A.C (1990) and expressed as $\mathrm{mg} / 100 \mathrm{~g}$ and titratable acidity determined in fruit juice as (\%) according to A.O.A.C (1990).

The experiment was arranged in split plot design with three replicates. Irrigation schedules were arranged in the main plots and plant densities were arranged in the sub main plots. Data were statistically analyzed using the analysis of variance method according to Snedicor and Cochran (1980). The means were compared by determining the least significant difference (L.S.D) at a probability level of $5 \%$.

\section{RESULTS}

The effect of irrigation scheduling and plant density on number of strawberry leaves was illustrated in Table (2). Data showed that irrigating strawberry plants for 15 min every 2 hours increased number of leaves comparing with the oth- er treatments. The differences between this treatment and the other were significant. Except irrigating plants for 15 min every $1 / 2$ hour. On the contrary, the system irrigated every 1 hour reduced number of leaves significantly. The reduction regarding number of leaves was significant in both seasons. On the other hand, data showed that there were no significant difference between PD-1 and PD-2 in the first season while it was significant in the second season.

Table 2. Effect of irrigation scheduling and plant density on number of strawberry leaves

\begin{tabular}{|c|c|c|c|c|c|}
\hline \multirow{2}{*}{$\begin{array}{l}\text { Irrigation } \\
\text { (Irri) }\end{array}$} & \multicolumn{4}{|c|}{ Plant Density (PD) } & \multirow{2}{*}{ Mean } \\
\hline & \multicolumn{2}{|c|}{ PD-1 } & \multicolumn{2}{|c|}{ PD-2 } & \\
\hline \multicolumn{6}{|c|}{ First season $(2013 / 2014)$} \\
\hline Cont & \multicolumn{2}{|c|}{11.63} & \multicolumn{2}{|c|}{12.03} & 11.83 \\
\hline Sched1 & \multicolumn{2}{|c|}{12.49} & \multicolumn{2}{|c|}{12.90} & 12.70 \\
\hline Sched2 & \multicolumn{2}{|c|}{8.88} & \multicolumn{2}{|c|}{9.19} & 9.04 \\
\hline Sched3 & \multicolumn{2}{|c|}{13.11} & \multicolumn{2}{|c|}{12.89} & 13.00 \\
\hline Sched4 & \multirow{2}{*}{\multicolumn{2}{|c|}{$\begin{array}{l}11.59 \\
11.54\end{array}$}} & \multirow{2}{*}{\multicolumn{2}{|c|}{$\begin{array}{l}11.89 \\
11.78\end{array}$}} & 11.74 \\
\hline Mean & & & & & \\
\hline \multicolumn{6}{|c|}{ Second season $(2014 / 2015)$} \\
\hline Cont & \multicolumn{2}{|c|}{9.17} & \multicolumn{2}{|c|}{9.50} & 9.34 \\
\hline Sched1 & \multicolumn{2}{|c|}{10.47} & \multicolumn{2}{|c|}{11.23} & 10.85 \\
\hline Sched2 & \multicolumn{2}{|c|}{6.83} & \multicolumn{2}{|c|}{7.50} & 7.17 \\
\hline Sched3 & \multicolumn{2}{|c|}{10.43} & \multicolumn{2}{|c|}{12.45} & 11.44 \\
\hline Sched4 & \multicolumn{2}{|c|}{11.77} & \multicolumn{2}{|c|}{12.17} & 11.97 \\
\hline Mean & \multicolumn{2}{|c|}{9.73} & \multicolumn{2}{|c|}{10.57} & \\
\hline \multicolumn{6}{|c|}{ L.S.D } \\
\hline \multicolumn{3}{|c|}{$\begin{array}{l}\text { First season } \\
(2013 / 2014)\end{array}$} & \multicolumn{3}{|c|}{$\begin{array}{c}\text { Second season } \\
(2014 / 2015)\end{array}$} \\
\hline Irri & PD & $\begin{array}{c}\text { Irri x } \\
\text { PD }\end{array}$ & Irri & PD & $\begin{array}{c}\text { Irri } \times \\
\text { PD }\end{array}$ \\
\hline 0.34 & N.S & 0.58 & 0.54 & 0.36 & 0.80 \\
\hline
\end{tabular}

Data for the interaction between irrigation scheduling and plant density showed that irrigating plants every 2 hours for 15 min combined with PD1 increased number of leaves followed by irrigation every 2 hours combined with PD-2 without a significant difference. While the irrigating plants every 1 hour combined with PD-1 reduced number of leaves. 
In the second season, similar results were obtained as the first season except that irrigating plants for 15 min every 3 hours increased number of leaves comparing with other treatments. The difference was significant comparing with the other treatments except irrigating every 2 hours for 15 min the difference was not significant. also, data showed that increase resulted from using PD2 was significant.

Data for the interaction between irrigation scheduling and plant density showed that irrigating plants every 2 hours combined with PD-2 increased number of leaves followed by irrigating every 3 hours combined with PD-2 without a significant difference between them. While the irrigation every 1 hour combined with PD-1 reduced number of leaves.

Table (3) presented the effect of irrigation scheduling and plant density on fruit set\%. Data showed that using Sched1 increased fruit set \% followed by control in the first season while in the second season the highest fruit set\% obtained using control followed by Sched 1 without significant difference in both seasons. The lowest fruit set $\%$ obtained using Sched 2.

Table 3. Effect of irrigation scheduling and plant density on fruit set \%

\begin{tabular}{|c|c|c|c|c|c|}
\hline \multirow{2}{*}{$\begin{array}{c}\text { Irrigation } \\
\text { (Irri) }\end{array}$} & \multicolumn{4}{|c|}{ Plant Density (PD) } & \multirow[b]{2}{*}{ Mean } \\
\hline & \multicolumn{2}{|c|}{ PD-1 } & \multicolumn{2}{|c|}{ PD-2 } & \\
\hline \multicolumn{6}{|c|}{ First season $(2013 / 2014)$} \\
\hline Cont & \multicolumn{2}{|c|}{95.89} & \multicolumn{2}{|c|}{94.68} & 95.28 \\
\hline Sched1 & \multicolumn{2}{|c|}{96.23} & \multicolumn{2}{|c|}{95.40} & 95.81 \\
\hline Sched2 & \multicolumn{2}{|c|}{80.73} & \multicolumn{2}{|c|}{83.61} & 82.17 \\
\hline Sched3 & \multicolumn{2}{|c|}{92.22} & \multicolumn{2}{|c|}{89.77} & 90.99 \\
\hline Sched4 & \multicolumn{2}{|c|}{91.54} & \multicolumn{2}{|c|}{89.85} & 90.69 \\
\hline Mean & \multicolumn{2}{|c|}{91.32} & \multicolumn{2}{|c|}{90.66} & \\
\hline \multicolumn{6}{|c|}{ Second season $(2014 / 2015)$} \\
\hline Cont & \multicolumn{2}{|c|}{91.92} & \multicolumn{2}{|c|}{93.86} & 92.89 \\
\hline Sched1 & \multicolumn{2}{|c|}{91.45} & \multicolumn{2}{|c|}{94.23} & 92.84 \\
\hline Sched2 & \multicolumn{2}{|c|}{58.54} & \multicolumn{2}{|c|}{80.67} & 69.61 \\
\hline Sched3 & \multicolumn{2}{|c|}{86.03} & \multicolumn{2}{|c|}{83.36} & 84.70 \\
\hline Sched4 & \multicolumn{2}{|c|}{88.21} & \multicolumn{2}{|c|}{91.43} & 89.82 \\
\hline Mean & \multicolumn{2}{|c|}{83.23} & \multicolumn{2}{|c|}{88.71} & \\
\hline \multicolumn{6}{|c|}{ L.S.D } \\
\hline \multicolumn{3}{|c|}{$\begin{array}{c}\text { First season } \\
(2013 / 2014)\end{array}$} & \multicolumn{3}{|c|}{$\begin{array}{c}\text { Second season } \\
(2014 / 2015)\end{array}$} \\
\hline Irri & PD & $\begin{array}{c}\text { Irri } \times \\
\text { PD }\end{array}$ & Irri & PD & $\begin{array}{c}\text { Irri } x \\
\text { PD }\end{array}$ \\
\hline 0.74 & N.S & 1.78 & 1.64 & 1.92 & 4.28 \\
\hline
\end{tabular}

On the contrary, data showed that, there was no significant difference between PD-1 and PD-2 in the first season while in the second season using PD-2 increased fruit set \% significantly.

Concerning the interaction between irrigation scheduling and plant density showed that sched1 combined with PD-followed by control 1 increased fruit set \% without a significant difference between them. The lowest fruit set \% obtained using Sched 2 combined with PD-1 in both seasons.

Data illustrated in Table (4 \& 5) presented the effect of irrigation scheduling and plant density on early \& total yield ( $\mathrm{g} / \mathrm{plant}$ ). Data showed that strawberry cultivated in the system using Sched1 recorded the highest early \& total yield followed by Sched 3 in the first season while in the second season using Sched 1 recorded the highest early yield followed by control. The lowest early \& total yield was obtained by Sched 2 . The differences among all treatments were significant.

Table 4. Effect of irrigation scheduling and plant density on early yield (g/plant)

\begin{tabular}{|c|c|c|c|c|c|}
\hline \multirow{2}{*}{$\begin{array}{l}\text { Irrigation } \\
\text { (Irri) }\end{array}$} & \multicolumn{4}{|c|}{ Plant Density (PD) } & \multirow{2}{*}{ Mean } \\
\hline & \multicolumn{2}{|c|}{ PD-1 } & \multicolumn{2}{|c|}{ PD-2 } & \\
\hline \multicolumn{6}{|c|}{ First season $(2013 / 2014)$} \\
\hline Cont & \multicolumn{2}{|c|}{208.00} & \multicolumn{2}{|c|}{255.00} & 231.50 \\
\hline Sched1 & \multicolumn{2}{|c|}{270.00} & \multicolumn{2}{|c|}{292.50} & 281.25 \\
\hline Sched2 & \multicolumn{2}{|c|}{152.00} & \multicolumn{2}{|c|}{173.70} & 162.85 \\
\hline Sched3 & \multicolumn{2}{|c|}{266.25} & \multicolumn{2}{|c|}{273.45} & 269.85 \\
\hline Sched4 & \multicolumn{2}{|c|}{191.68} & \multicolumn{2}{|c|}{244.44} & 218.06 \\
\hline Mean & \multicolumn{2}{|c|}{217.59} & \multicolumn{2}{|c|}{247.82} & \\
\hline \multicolumn{6}{|c|}{ Second season $(2014 / 2015)$} \\
\hline Cont & \multicolumn{2}{|c|}{136.50} & \multicolumn{2}{|c|}{198.00} & 167.25 \\
\hline Sched1 & \multicolumn{2}{|c|}{150.62} & \multicolumn{2}{|c|}{300.00} & 225.31 \\
\hline Sched2 & \multicolumn{2}{|c|}{28.00} & \multicolumn{2}{|c|}{66.50} & 47.25 \\
\hline Sched3 & \multicolumn{2}{|c|}{108.50} & \multicolumn{2}{|c|}{135.00} & 121.75 \\
\hline Sched4 & \multicolumn{2}{|c|}{140.07} & \multicolumn{2}{|c|}{160.33} & 150.20 \\
\hline Mean & \multicolumn{2}{|c|}{112.74} & \multicolumn{2}{|c|}{171.97} & \\
\hline \multicolumn{6}{|c|}{ L.S.D } \\
\hline \multicolumn{3}{|c|}{$\begin{array}{c}\text { First season } \\
(2013 / 2014)\end{array}$} & \multicolumn{3}{|c|}{$\begin{array}{c}\text { Second season } \\
(2014 / 2015)\end{array}$} \\
\hline Irri & PD & $\begin{array}{c}\text { Irri } \times \\
\text { PD }\end{array}$ & Irri & PD & $\begin{array}{c}\text { Irri } \times \\
\text { PD }\end{array}$ \\
\hline 3.15 & 2.11 & 4.72 & 1.79 & 1.59 & 3.56 \\
\hline
\end{tabular}



strawberry grown in water culture

Table 5. Effect of irrigation scheduling and plant density on total yield ( $\mathrm{g} / \mathrm{plant})$

\begin{tabular}{|c|c|c|c|c|c|}
\hline \multirow{2}{*}{$\begin{array}{c}\text { Irrigation } \\
\text { (Irri) }\end{array}$} & \multicolumn{4}{|c|}{ Plant Density (PD) } & \multirow{2}{*}{ Mean } \\
\hline & \multicolumn{2}{|c|}{ PD-1 } & \multicolumn{2}{|c|}{ PD-2 } & \\
\hline \multicolumn{6}{|c|}{ First season (2013/2014) } \\
\hline Cont & \multicolumn{2}{|c|}{760.50} & \multicolumn{2}{|c|}{840.00} & 800.25 \\
\hline Sched1 & \multicolumn{2}{|c|}{945.75} & \multicolumn{2}{|c|}{954.00} & 949.88 \\
\hline Sched2 & \multicolumn{2}{|c|}{636.50} & \multicolumn{2}{|c|}{707.35} & 671.92 \\
\hline Sched3 & \multicolumn{2}{|c|}{834.25} & \multicolumn{2}{|c|}{865.93} & 850.09 \\
\hline Sched4 & \multicolumn{2}{|c|}{676.87} & \multicolumn{2}{|c|}{780.85} & \multirow[t]{2}{*}{728.86} \\
\hline Mean & \multicolumn{2}{|c|}{770.77} & \multicolumn{2}{|c|}{829.62} & \\
\hline \multicolumn{6}{|c|}{ Second season $(2014 / 2015)$} \\
\hline Cont & \multicolumn{2}{|c|}{714.00} & \multicolumn{2}{|c|}{836.00} & 775.00 \\
\hline Sched1 & \multicolumn{2}{|c|}{725.70} & \multicolumn{2}{|c|}{918.75} & 822.22 \\
\hline Sched2 & \multicolumn{2}{|c|}{406.00} & \multicolumn{2}{|c|}{448.88} & 427.44 \\
\hline Sched3 & \multicolumn{2}{|c|}{527.00} & \multicolumn{2}{|c|}{666.00} & 596.50 \\
\hline Sched4 & \multicolumn{2}{|c|}{662.13} & 764 & & 713.40 \\
\hline Mean & 60 & .97 & 726. & & \\
\hline & & L.S. & & & \\
\hline $\begin{array}{l}\text { Firs } \\
(20\end{array}$ & $\begin{array}{l}\text { seaso } \\
/ 2014\end{array}$ & & & $\begin{array}{l}\text { ind se } \\
14 / 20\end{array}$ & $\begin{array}{l}\text { eason } \\
\text { D15) }\end{array}$ \\
\hline Irri & PD & $\begin{array}{c}\text { Irri } \times \\
\text { PD }\end{array}$ & Irri & PD & $\begin{array}{c}\text { Irri x } \\
\text { PD }\end{array}$ \\
\hline 13.13 & 6.02 & 13.47 & 4.56 & 5.57 & 12.45 \\
\hline
\end{tabular}

On the other hand, plants cultivated in PD-2 gave the highest early\& total yield comparing with PD-1 significantly in both seasons.

Data for the interaction between irrigation scheduling and plant density showed that plants cultivated using Sched 1combined with PD-2 gave the highest early yield followed by the system irrigated every 2 hours in PD-2 significantly in the first season while in the second season the system irrigated every $1 / 2$ hour recorded the highest early yield followed by control significantly. The lowest early yield was obtained by the plants cultivated in Sched 2 combined with PD-1significantly in both seasons.

Regarding mean weight of strawberry fruits, data in Table (6) showed that the using Sched 1 increased mean fruit weight followed by Sched 3 without significant difference. On the contrary, using Sched 2 reduced mean fruit weight significantly.
On the other hand, plants cultivated using PD-2 increased mean fruit weight significantly comparing with PD-1.

Data for the interaction between irrigation scheduling and plant density showed that plants cultivated using Sched 1combined with PD-2 increased mean fruit weight followed by Sched 3 combined with PD-2 significantly. The lowest mean fruit weight obtained by the plant cultivated using Sched 2 combined PD-1.

Table 6. Effect of irrigation scheduling and plant density on mean fruit weight $(\mathrm{g})$

\begin{tabular}{|c|c|c|c|c|c|}
\hline \multirow{2}{*}{$\begin{array}{l}\text { Irrigation } \\
\text { (Irri) }\end{array}$} & \multicolumn{4}{|c|}{ Plant Density (PD) } & \multirow{2}{*}{ Mean } \\
\hline & \multicolumn{2}{|c|}{ PD-1 } & \multicolumn{2}{|c|}{ PD-2 } & \\
\hline \multicolumn{6}{|c|}{ First season $(2013 / 2014)$} \\
\hline Cont & \multicolumn{2}{|c|}{13.00} & \multicolumn{2}{|c|}{15.00} & 14.00 \\
\hline Sched1 & \multicolumn{2}{|c|}{18.00} & \multicolumn{2}{|c|}{19.50} & 18.75 \\
\hline Sched2 & \multicolumn{2}{|c|}{9.50} & \multicolumn{2}{|c|}{9.65} & 9.58 \\
\hline Sched3 & \multicolumn{2}{|c|}{17.75} & \multicolumn{2}{|c|}{18.23} & 17.99 \\
\hline Sched4 & \multicolumn{2}{|c|}{11.98} & \multicolumn{2}{|c|}{13.58} & 12.78 \\
\hline Mean & \multicolumn{2}{|c|}{14.05} & \multicolumn{2}{|c|}{15.19} & \\
\hline \multicolumn{6}{|c|}{ Second season $(2008 / 2009)$} \\
\hline Cont & \multicolumn{2}{|c|}{10.51} & \multicolumn{2}{|c|}{11.00} & 10.76 \\
\hline Sched1 & \multicolumn{2}{|c|}{13.70} & \multicolumn{2}{|c|}{18.78} & 16.24 \\
\hline Sched2 & \multicolumn{2}{|c|}{7.03} & \multicolumn{2}{|c|}{8.31} & 7.67 \\
\hline Sched3 & \multicolumn{2}{|c|}{7.77} & \multicolumn{2}{|c|}{9.00} & 8.38 \\
\hline Sched4 & \multicolumn{2}{|c|}{12.76} & \multicolumn{2}{|c|}{12.35} & 12.56 \\
\hline Mean & \multicolumn{2}{|c|}{10.35} & \multicolumn{2}{|c|}{11.89} & \\
\hline \multicolumn{6}{|c|}{ L.S.D } \\
\hline \multicolumn{3}{|c|}{$\begin{array}{c}\text { First season } \\
(2013 / 2014)\end{array}$} & \multicolumn{3}{|c|}{$\begin{array}{c}\text { Second season } \\
(2014 / 2015)\end{array}$} \\
\hline Irri & PD & $\begin{array}{c}\text { Irri } \times \\
\text { PD }\end{array}$ & Irri & PD & $\begin{array}{c}\text { Irri } \times \\
\text { PD }\end{array}$ \\
\hline 1.07 & 0.54 & 1.22 & 0.19 & 0.28 & 0.62 \\
\hline
\end{tabular}

Table (7) illustrated the effect of irrigation scheduling and plant density on total soluble solids (TSS) \%. Data showed that strawberry plants cultivated using Sched 1 increased TSS \% in the fruit significantly in both seasons.

On the other hand, there were no significant difference between PD-1 and PD-2 in both seasons. 
Data for the interaction between irrigation scheduling and plant density showed that plants cultivated Sched 1 combined with PD-2 increased TSS $\%$ followed by the same Irrigation scheduling combined with PD-1without significant difference between them. The difference Sched 1 combined with PD-2 was significant comparing with all other treatments.

In the second season, data for the interaction between irrigation scheduling and plant density showed that plants cultivated using Sched 1 combined with PD-1 increased TSS \% followed by irrigation every 3 hours combined with PD- 1 followed by irrigation every $1 / 2$ hour combined with PD-2 without a significant difference between them. The lowest TSS obtained by the plant cultivated in system irrigated every 1 hour combined with PD-1.

Table 7. Effect of irrigation scheduling and plant density on Total Soluble Solids (TSS) \%

\begin{tabular}{|c|c|c|c|c|c|}
\hline \multirow{2}{*}{$\begin{array}{c}\text { Irrigation } \\
\text { (Irri) }\end{array}$} & \multicolumn{4}{|c|}{ Plant Density (PD) } & \multirow[b]{2}{*}{ Mean } \\
\hline & \multicolumn{2}{|c|}{ PD-1 } & PD- & & \\
\hline \multicolumn{6}{|c|}{ First season (2013/2014) } \\
\hline Cont & \multicolumn{2}{|c|}{6.23} & \multicolumn{2}{|c|}{6.33} & 6.28 \\
\hline Sched1 & \multicolumn{2}{|c|}{6.90} & \multicolumn{2}{|c|}{7.10} & 7.00 \\
\hline Sched2 & \multicolumn{2}{|c|}{5.50} & \multicolumn{2}{|c|}{5.80} & 5.65 \\
\hline Sched3 & \multicolumn{2}{|c|}{6.23} & \multicolumn{2}{|c|}{6.07} & 6.15 \\
\hline Sched4 & \multicolumn{2}{|c|}{6.60} & \multicolumn{2}{|c|}{5.93} & 6.27 \\
\hline Mean & \multicolumn{2}{|c|}{6.29} & \multicolumn{2}{|c|}{6.25} & \\
\hline \multicolumn{6}{|c|}{ Second season $(2014 / 2015)$} \\
\hline Cont & \multicolumn{2}{|c|}{5.57} & \multicolumn{2}{|c|}{5.80} & 5.69 \\
\hline Sched1 & \multicolumn{2}{|c|}{6.60} & \multicolumn{2}{|c|}{6.20} & 6.40 \\
\hline Sched2 & \multicolumn{2}{|c|}{5.17} & \multicolumn{2}{|c|}{5.23} & 5.20 \\
\hline Sched3 & \multicolumn{2}{|c|}{6.13} & \multicolumn{2}{|c|}{5.90} & 6.02 \\
\hline Sched4 & \multicolumn{2}{|c|}{6.43} & \multicolumn{2}{|c|}{5.90} & 6.17 \\
\hline Mean & \multicolumn{2}{|c|}{5.98} & \multicolumn{2}{|c|}{5.81} & \\
\hline \multicolumn{6}{|c|}{ L.S.D } \\
\hline \multicolumn{3}{|c|}{$\begin{array}{c}\text { First season } \\
(2013 / 2014)\end{array}$} & \multicolumn{3}{|c|}{$\begin{array}{c}\text { Second season } \\
(2014 / 2015)\end{array}$} \\
\hline Irri & PD & $\begin{array}{c}\text { Irri } x \\
\text { PD }\end{array}$ & Irri & PD & $\begin{array}{c}\text { Irri } \times \\
\text { PD }\end{array}$ \\
\hline 0.26 & N.S & 0.40 & 0.15 & N.S & 0.44 \\
\hline
\end{tabular}

Data illustrated in Table (8) presented the effect of irrigation scheduling and plant density on vitamin C content in the fruit $(\mathrm{mg} / 100 \mathrm{~g}$ fresh weight), Data showed that strawberry cultivated using irrigation every 1 hour increased vitamin C content in the fruit significantly while strawberry plants cultivated using irrigation every 2 hours did not affect vitamin $\mathrm{C}$ content significantly.

On the other hand, plants cultivated in PD-2 increased vitamin $\mathrm{C}$ content in the fruits significantly comparing with PD-1.

Data for the interaction between irrigation scheduling and plant density showed that plants cultivated using irrigation every 1 hour companied with PD-2 increased vitamin C content in the fruit significantly. While the strawberry plants cultivated using irrigation every 2 hours combined with PD-1 decreased vitamin $\mathrm{C}$ content significantly.

Table 8. Effect of irrigation scheduling and plant density on Vitamin C (mg/100 g fresh weight)

\begin{tabular}{|c|c|c|c|c|c|}
\hline \multirow{2}{*}{$\begin{array}{c}\text { Irrigation } \\
\text { (Irri) }\end{array}$} & \multicolumn{4}{|c|}{ Plant Density (PD) } & \multirow{2}{*}{ Mean } \\
\hline & \multicolumn{2}{|c|}{ PD-1 } & \multicolumn{2}{|c|}{ PD-2 } & \\
\hline \multicolumn{6}{|c|}{ First season $(2013 / 2014)$} \\
\hline Cont & \multicolumn{2}{|c|}{110.00} & \multicolumn{2}{|c|}{100.00} & 105.00 \\
\hline Sched1 & \multicolumn{2}{|c|}{96.00} & \multicolumn{2}{|c|}{110.00} & 103.00 \\
\hline Sched2 & \multicolumn{2}{|c|}{109.00} & \multicolumn{2}{|c|}{115.00} & 112.00 \\
\hline Sched3 & \multicolumn{2}{|c|}{93.00} & \multicolumn{2}{|c|}{94.00} & 93.50 \\
\hline Sched4 & \multicolumn{2}{|c|}{103.67} & \multicolumn{2}{|c|}{104.33} & 104.00 \\
\hline Mean & \multicolumn{2}{|c|}{102.3} & \multicolumn{2}{|c|}{104.67} & \\
\hline \multicolumn{6}{|c|}{ Second season $(2014 / 2015)$} \\
\hline Cont & \multicolumn{2}{|c|}{130.00} & \multicolumn{2}{|c|}{140.00} & 135.00 \\
\hline Sched1 & \multicolumn{2}{|c|}{102.33} & \multicolumn{2}{|c|}{110.00} & 106.17 \\
\hline Sched2 & \multicolumn{2}{|c|}{105.00} & \multicolumn{2}{|c|}{120.00} & 112.50 \\
\hline Sched3 & \multicolumn{2}{|c|}{100.00} & \multicolumn{2}{|c|}{96.00} & 98.00 \\
\hline Sched4 & \multicolumn{2}{|c|}{100.00} & \multicolumn{2}{|c|}{110.00} & \multirow[t]{2}{*}{105.00} \\
\hline Mean & 107 & .47 & 115.2 & & \\
\hline \multicolumn{6}{|c|}{ L.S.D } \\
\hline \multicolumn{3}{|c|}{$\begin{array}{l}\text { First season } \\
(2013 / 2014)\end{array}$} & $\mathrm{Sec}$ & $\begin{array}{l}\text { ond se } \\
014 / 20\end{array}$ & $\begin{array}{l}\text { eason } \\
15)\end{array}$ \\
\hline Irri & PD & $\begin{array}{c}\text { Irri } \times \\
\text { PD }\end{array}$ & Irri & PD & $\begin{array}{c}\text { Irri } \times \\
\text { PD }\end{array}$ \\
\hline 3.58 & 1.94 & 4.33 & 3.76 & 1.75 & 3.91 \\
\hline
\end{tabular}



strawberry grown in water culture

In the second season, Data showed that strawberry cultivated in control recorded the highest vitamin $\mathrm{C}$ content in the fruit significantly.

Data illustrated in Table (9) presented the effect of irrigation scheduling and plant density on fruit acidity, Data showed that strawberry cultivated using Sched 3 recorded the highest value of fruit acidity followed by control, while the lowest value of fruit acidity was obtained by the plants cultivated using Sched 4 significantly.

Table 9. Effect of irrigation scheduling and plant density on fruit acidity

\begin{tabular}{|c|c|c|c|c|c|}
\hline \multirow{2}{*}{\multicolumn{2}{|c|}{$\begin{array}{c}\text { Irrigation } \\
\text { (Irri) } \\
\end{array}$}} & \multicolumn{3}{|c|}{ Plant Density (PD) } & \multirow[b]{2}{*}{ Mean } \\
\hline & & PD-1 & \multicolumn{2}{|c|}{ PD-2 } & \\
\hline \multicolumn{6}{|c|}{ First season $(2013 / 2014)$} \\
\hline \multicolumn{2}{|c|}{ Cont } & 1.05 & \multicolumn{2}{|c|}{1.02} & 1.04 \\
\hline \multicolumn{2}{|c|}{ Sched1 } & 0.74 & \multicolumn{2}{|c|}{0.77} & 0.76 \\
\hline \multicolumn{2}{|c|}{ Sched2 } & 0.83 & \multicolumn{2}{|c|}{0.72} & 0.78 \\
\hline \multicolumn{2}{|c|}{ Sched3 } & 0.85 & \multicolumn{2}{|c|}{1.27} & 1.06 \\
\hline \multicolumn{2}{|c|}{ Sched4 } & 0.83 & \multicolumn{2}{|c|}{0.49} & 0.66 \\
\hline \multicolumn{2}{|c|}{ Mean } & 0.86 & \multicolumn{2}{|c|}{0.85} & \\
\hline \multicolumn{6}{|c|}{ Second season $(2014 / 2015)$} \\
\hline \multicolumn{2}{|c|}{ Cont } & 0.99 & \multicolumn{2}{|c|}{1.05} & 1.02 \\
\hline \multicolumn{2}{|c|}{ Sched1 } & 0.77 & \multicolumn{2}{|c|}{0.75} & 0.76 \\
\hline \multicolumn{2}{|c|}{ Sched2 } & 0.82 & \multicolumn{2}{|c|}{0.82} & 0.82 \\
\hline \multicolumn{2}{|c|}{ Sched3 } & 0.46 & \multicolumn{2}{|c|}{1.37} & 0.92 \\
\hline \multicolumn{2}{|c|}{ Sched4 } & 0.86 & \multicolumn{2}{|c|}{0.45} & 0.66 \\
\hline \multicolumn{2}{|c|}{ Mean } & 0.78 & \multicolumn{2}{|c|}{0.89} & \\
\hline \multicolumn{6}{|c|}{ L.S.D } \\
\hline \multicolumn{3}{|c|}{$\begin{array}{c}\text { First season } \\
(2013 / 2014)\end{array}$} & \multicolumn{3}{|c|}{$\begin{array}{c}\text { Second season } \\
(2014 / 2015)\end{array}$} \\
\hline Irri & PD & $\begin{array}{c}\text { Irri } \times \\
\text { PD }\end{array}$ & Irri & PD & $\begin{array}{c}\text { Irri } \times \\
\text { PD }\end{array}$ \\
\hline 0.03 & N.S & 0.12 & 0.05 & 0.03 & 0.07 \\
\hline
\end{tabular}

On the other hand, there were no significant difference between PD-1 and PD-2.

Data for the interaction between irrigation scheduling and plant density showed that plants cultivated using irrigation every 2 hours combined with PD-2 recorded the highest value of fruit acidity significantly. While the strawberry plants cultivated irrigation every 3 hours combined with PD-2 recorded the lowest value of fruit acidity significantly.
In the second season, Data showed that strawberry cultivated control recorded the highest value of fruit acidity followed by irrigation every 2 hours while the lowest value of fruit acidity was obtained by the plants irrigated every 3 hours. There were significant differences among all treatments.

On the other hand, plants cultivated in PD-2 gave the highest value of fruit acidity significantly comparing with PD-1.

Data for the interaction between irrigation scheduling and plant density showed that plants irrigated every 2 hours combined with PD-2 recorded the highest value of fruit acidity significantly. While the strawberry plants irrigated every 3 hours combined with PD-2 recorded the lowest value of fruit acidity.

\section{DISCUSSION}

From the overall results it clear that irrigating strawberry plants for 15 min every $1 / 2$ hour increased early \& total yield. This is could resulted from increasing number of leaves as obtained from the results of this study leading to increase the vegetative growth. The increase of vegetative growth increase the photosynthesis leading to increase fruit set $\%$ and mean fruit weight. This resulted to increase early and total yield. The increase of vegetative growth leads to more yields as suggested by Sandwell (1977), Maher (1980), Moss (1983), Moorby and Graves (1980), Tankano (1988), Economakis, (1992) and Economakis and Krulj (2001). Bish-EB et al (1997) and Hennion et al (1997) found that bigger plants produced higher yield resulted from higher leaf area that increased the photosynthesis, which reflected on the yield.

Regarding planting density, data showed that planting density plays an important role in achieving high productivity per unit area. High planting density resulted in highest yield, largest berries, and the best quality fruit (Petersen, 1998). In strawberry, marketable yields were higher at narrower spacing than wider spacing (Legard et al 2000).

Concerning the effect of the interaction irrigating strawberry plants for 15 min every $1 / 2$ hour combined with high density of plants gave the highest mean fruit weight, early yield, total yield. This is resulted from combining the best treatment for irrigation scheduling with the best plant density leading to high early and total yield as discussed before. 


\section{CONCLUSION}

For the overall results it clear that the system irrigated every $1 / 2$ hour increased mean fruit weight, early \& total yield and TSS but reduced vitamin C and fruit acidity. Also, plants cultivated in PD-2 gave the highest mean fruit weight, early\& total yield, vitamin $\mathrm{C}$ and fruit acidity comparing with PD-1 but not affected on TSS. The system irrigated every $1 / 2$ hour in PD-2 gave the highest mean fruit weight, early yield, total yield and TSS.

\section{REFERENCES}

A.O.A.C. 1990. Official Methods of Analysis of the Association Official Analytical Chemists. 15 ${ }^{\text {th }}$, ed. Published by the Association of Official Analytical Chemists, Inc. Anlington, Virginia 22201, U.S.A.

Bish, EB, Cantliffe, D.J., Hochmuth, G.J., Chandler, C.K. 1997. Development of containerized strawberry transplants for Florida's winter production system. Acta Hort., 439, 469-465.

De-Camacaro, M.E.P., Camacaro, G.J., Hadley, P., Dennett, M.D., Battey, N.H. and Carew, J.G. 2004. Effect of plant density and initial crown size on growth, development and yield in strawberry cultivars Elsanta and Bolero. J. Hort. Sci. and Biotech. 79(5), 739-746.

Economakis, C.D. 1992. The influence of solution heating and intermittent solution circulation on tomatoes in nutrient film culture. Acta Hort. 323, 81-87.

Economakis, C.D, and Krulj, L. 2001. Effect of root - zone warming on strawberry plants grown with Nutrient Film Technique (NFT). Acta Hort. 548, 189-196.

El-Behairy, 1994. The effect of levels of phosphorus and zinc in the nutrient solution on macro and micronutrients uptake and translocation in cucumber (Cucumus sativus L.) grown by the nutrient film technique. Ph.D. Thesis, London University, 299 p.

Hakkinen, S.H. and Torronen. A.R. 2000. Content of flavonols and selected phenolic acids in strawberries and Vaccinium species: influence of cultivar, cultivation site and technique. Food Res. Int. 33, 517-524.

Hennion, B., Schupp, J., Longuesserre, J, 1997. Fraisimotte $R$ a strawberry plug plant developed by CIREF in France. Acta Hort., 439, 469-474.
Hoberg, E., Ulrich, D., Krüger, E. and Schöpplein. E. 2002. Effect of irrigation on strawberry flavour quality. Proc. $4^{\text {th }}$ Intl. Strawberry Symp., pp. 735-736.

Jansen, W.A.G.M. 1997. Growing media and plant densities for strawberry tray plants. Acta Hort. 439, 457-460.

Kirnak, H., Kaya, C., Higgs, D., Bolat, I., Simsek, M. and Ikinci. A. 2003. Effects of preharvest drip-irrigation scheduling on strawberry yield, quality and growth. Austral. J. Experimental Agr., 43, 105-111.

Krüger, E., Schmidt, G. and Brückner. U. 1999. Scheduling strawberry irrigation based upon tensiometer easurement and a climatic water balance model. Scientia Hort. 81, 409-424.

Legard, D.E., Xiao, C.L., Mertely, J.C. and Chandler. C.K. 2000. Effects of plant spacing and cultivar on incidence of botrytis fruit rot in annual strawberry. University of Florida, Gulf Coast Research and Education Center, Lewis Gallagher Road, Dover. 84(5), 531-538.

Maher, M.J. 1980. Effect of root zone warming, flow rate and propagation system on tomato crop performance in N.F.T. Acta Horticulturae, 98, 46-52.

Moorby, J. and Graves, C.J. 1980. Root and air temperature effects on growth and yield of tomatoes and lettuce. Acta Hort., 98, 29-43.

Moss, G.J. 1983. Root -zone warming of greenhouse tomatoes in nutrient film as a means of reducing heating requirements. J. Hort. Sci., 58(1), 103-109.

Petersen, V.O. 1998. Influence of raised beds, plant density, and fertigation on yield and quality of strawberry (Fragaria $\times$ Ananassa Duch.). Europ. J. Hort. Sci., 63(1), 19-22.

Sandwell, I., 1977. Warm rooted tomatoes crop satisfactorily at $\stackrel{C}{C}$ night. Grower. 88(13), 605.

Sharma, R.R. 2002. Growing strawberry. Int. Book Distributing Co., India. 1, 1-2.

Sharma, V.P. and Sharma, R.R. 2004. The strawberry. Indian Council of Agricultural Research (ICAR) NEW DELHI, INDIA.

Simonne, E.H. and Dukes. M.D. 2009. Principles and practices of irrigation management for vegetables, pp. 17-23. In: S.M. Olson and E.H. Simonne (eds.). Vegetable production handbook for Florida, 2009-2010. IFAS Publ., Univ. of Florida. 
Snedicor, R. and Cochran, W. 1980. Statistical methods. Sixth edition, lowa State Univ. Press, Amer. lowa, USA.

Sturm, K., Koron, D. and Stampar, F. 2003. The composition of fruit of different strawberry varieties depending on maturity stage. J. Food Chem., 83, 417-422.
Takeda, F. 2000. Out-of-season greenhouse strawberry production in soilless substrate. Adv. Strawberry Res., 18, 4-15.

Tanko, T. 1988. Effect of conductivity and temperature of nutrient solution on the mineral nutrition of horticultural crops in water culture. Acta Horticulturae 230, 299-305. 